\title{
Random-exchange quantum Heisenberg antiferromagnets on the square lattice
}

\author{
Nicolas Laflorencie, ${ }^{1}$ Stefan Wessel, ${ }^{2}$ Andreas Läuchli, ${ }^{3}$ and Heiko Rieger ${ }^{4}$ \\ ${ }^{1}$ Department of Physics \& Astronomy, University of British Columbia, Vancouver, B.C., Canada, V6T 1Z1 \\ ${ }^{2}$ Institut für Theoretische Physik III, Universität Stuttgart, 70550 Stuttgart, Germany \\ ${ }^{3}$ Institut Romand de Recherche Numérique en Physique des Matériaux, EPFL, 1015 Lausanne, Switzerland \\ ${ }^{4}$ Theoretische Physik; Universität des Saarlandes, 66041 Saarbrücken; Germany
}

(Dated: November 7, 2018)

\begin{abstract}
The ground state properties of random-exchange spin- $1 / 2$ Heisenberg antiferromagnets on the square lattice are investigated using a combination of quantum Monte Carlo simulations, exact numerical diagonalizations, and spin wave calculations. Whereas arbirarily weak disorder has a drastic effect on 1d Heisenberg AFM, we find that in two dimensions the characteristics of the ground state like long-range order is robust even against strong disorder. While the antiferromagnetic order parameter and the spin stiffness are exponentially reduced for singular exchange distributions, they vanish only in the limit of infinite randomness.
\end{abstract}

PACS numbers: $75.40 . \mathrm{Mg}, 75.10 . \mathrm{Nr}, 02.70 . \mathrm{Ss}, 75.30 . \mathrm{Ds}$

The spin- $1 / 2$ Heisenberg antiferromagnet (AFM) on the square lattice has attracted a lot of interest in the last two decades 1, 2, 3, 4, 5, 6, 7, 8], motivated in particular by the suggestion that the high-temperature superconductivity in cuprates is related to the magnetic properties of their $\mathrm{CuO}$ planes 9]. Its ground state (GS) is antiferromagnetically ordered and the value for the staggered magnetization $m_{\mathrm{AF}}=0.3070(3)$ is known to high accuracy [8]. Recently it was found that this magnetic long range order (LRO) is robust against the introduction of static non-magnetic impurities, as observed in $\mathrm{Mg}$ or $\mathrm{Zn}$ doped $\mathrm{La}_{2} \mathrm{CuO}_{4}$ [10]. In fact, using numerical simulations, it was shown that LRO persists up to the percolation threshold for site dilution 11, 12]. Furthermore, the critical exponents for the transition to the paramagnetic phase are those of the classical percolation transition 12.

The question then arises, how the GS properties change for other forms of quenched disorder, in particular for generic bond disorder, i.e. randomness in the strength of the antiferromagnetic exchange coupling. In the one-dimensional (1D) case, where the GS of the pure system is critical, bond disorder is relevant [13], and an infinitesimal amount of bond disorder drives the system into the random singlet phase with unconventional scaling properties [14, 15, 16]. On the other hand, the gapped spin-liquid GS of antiferromagnetic spin- $1 / 2$ Heisenberg ladders is stable against the introduction of bond disorder [17, 18]. Indications for similar stability were obtained recently also for two-dimensional (2D) Heisenberg antiferromagnets using the strong disorder renormalization group 19, 20], which is, however, reliable only for the opposite scenario, i.e. when the GS is described by an infinite randomness fixed point scenario.

Here, we employ exact numerical methods to reliably estimate the stability of the GS of 2D Heisenberg AFMs against exchange-randomness. In particular, we consider the bond-disordered spin- $1 / 2$ Heisenberg AFM on the square lattice, defined by the Hamiltonian

$$
\mathcal{H}=\sum_{\langle i, j\rangle} J_{i j} \mathbf{S}_{i} \cdot \mathbf{S}_{j},
$$

where $\langle i, j\rangle$ are nearest neighbor bonds on a $L \times L$ square lattice $\left(N=L^{2}\right), \mathbf{S}_{i}$ are spin-1/2 operators, and the AFM exchange couplings $J_{i j} \geq 0$ are quenched random variables with probability distribution $\mathcal{P}(J)$. In the following, we will consider two different types of disorder distributions: (i) a $W$-dependent flat, bounded bond distribution, where the bonds are uniformly and symmetrically distributed around 1 with width $W$ :

$$
\mathcal{P}(J)=\Theta(J-(1-W)) \cdot \Theta((1+W)-J) / 2 W
$$

and (ii) a singular distribution controlled by the parameter $\delta$ and given by [21]

$$
\mathcal{P}(J)=J^{-1+\delta^{-1}} \delta^{-1} \cdot \Theta(J) \Theta(1-J) .
$$

This second type of disorder distribution allows us to study the stability of the GS towards a singular proliferation of weak bonds in the lattice structure.

We analyze the GS properties of the model (11) using a combination of quantum Monte Carlo (QMC) simulations, exact diagonalizations (ED) based on the Lanczos method, and spin wave theory (SW). In particular, we consider the stability of the staggered magnetization and the spin stiffness towards bond disorder, using disorder averaged quantities as obtained from several thousand disorder realizations from the distributions (2) and (3) with different parameters $W$ or $\delta$, respectively.

A Néel-ordered GS in the thermodynamic limit is signaled by a non-vanishing infinite system size limit $m_{\mathrm{AF}}^{2}$ of the staggered structure factor per site $s(\pi, \pi)$ :

$$
s(\pi, \pi)=\frac{3}{N^{2}}\left\langle\left(\sum_{i=1}^{N}(-1)^{i} S_{i}^{z}\right)^{2}\right\rangle \rightarrow m_{\mathrm{AF}}^{2} \quad(N \rightarrow \infty) .
$$




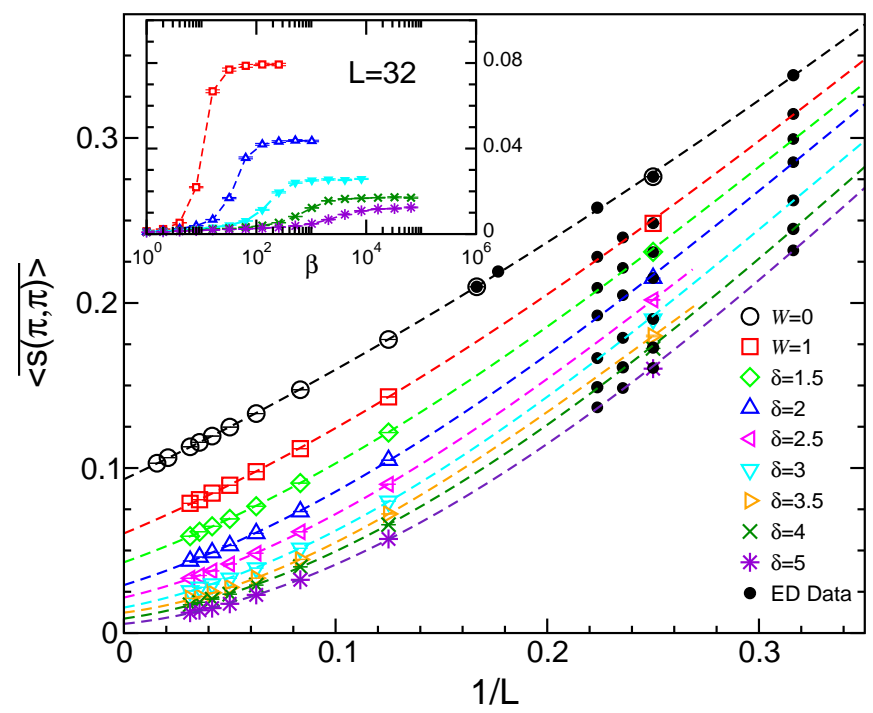

FIG. 1: (Color online) Disorder averaged value of the GS staggered structure factor $\overline{\langle s(\pi, \pi)\rangle}$ as a function of $1 / L$. Filled symbols are ED data and open symbols QMC data. The number of random samples varied between 1000 for the largest lattice and 10000 for the smaller lattices. Dashed lines denote third order polynomial fits of the finite size data. The inset exhibits the convergence of $\langle s(\pi, \pi)\rangle$ using $\beta$-doubling scheme for $\delta=1,2,3,4,5$ (top to bottom) and $L=32$.

The QMC simulations have been performed using the stochastic series expansion technique with a directed loop update scheme [22]. For the clean (i.e. no disorder) case, we studied square lattices up to $L=64$. We found that a scaling of the inverse temperature as $\beta=8 \times L$ allows to obtain GS properties. For the random case we needed to perform the QMC simulations at much lower temperature, as correlations on weak bonds (i.e. small $J_{i j}$ ) still develop at very low temperatures. To stimate GS quantities reliable, we used the $\beta$-doubling scheme 12] which accelerates the simulations considerably at low temperatures. We were able to extract the GS properties of systems with up to $32 \times 32$ lattice sites, for values of the disorder-parameter up to $\delta=5$ by doubling $\beta$ up to $2^{16}$ (see the inset of Fig. 11). For the small lattices we have also carefully checked the convergence against the ED results. The number of random samples considered for disorder averaged quantities varied between 1000 for the largest lattice and 10000 for smaller lattices.

In Fig. 1 1 we show the disorder averaged GS values of $\overline{\langle s(\pi, \pi)\rangle}$, which we obtained from the QMC and ED calculations as functions of $1 / L$. For the clean case $(\delta=0)$ QMC data for lattices ranging from $L=4$ up to $L=64$ have been extrapolated to the thermodynamic limit using an unconstrained third order polynomial fit in $1 / L$. The obtained staggered magnetization $m_{\mathrm{AF}}=0.3064(2)$, is in good agreement with previous estimates [8, 23. Increasing the randomness $\delta$, we found that large system

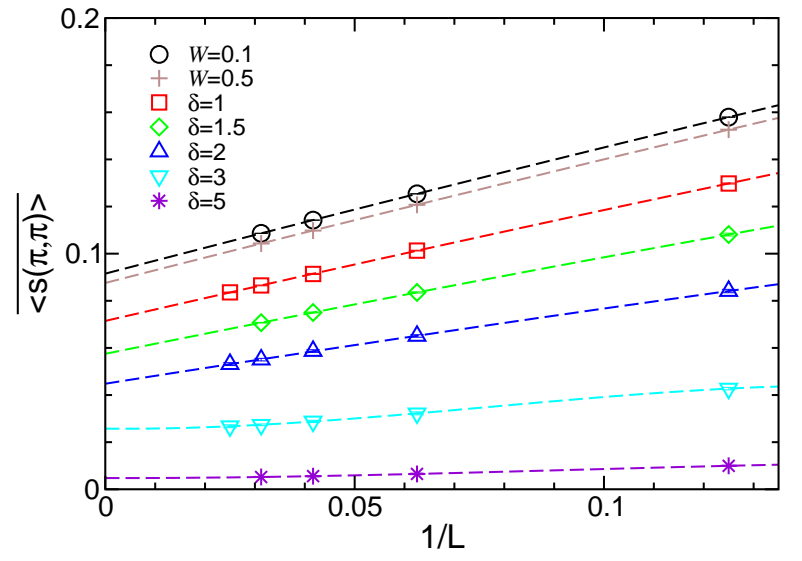

FIG. 2: (Color online) Results from spin wave theory for the disorder averaged value of the GS staggered structure factor $\overline{\langle s(\pi, \pi)\rangle}$ as a function of $1 / L$. Dashed lines denote polynomial fits in $1 / L$.

sizes are required to reliably extrapolate to the thermodynamic limit. While in the clean case and for weak disorder the finite size scaling is dominated by a term linear in $1 / L$, higher-order terms become important for larger values of $\delta$. This can be seen from Fig. [1 where for strong disorder and large systems sizes, the curvature of the polynomial extrapolations become more pronounced, indeed requiring up to third order polynomials in $1 / L$. We checked that the extrapolated values did not change within the statistical error bars upon allowing higher order fitting polynomials.

To further analyze the effects of the randomness on the quantum fluctuations above the classical Néel state, we employ linear spin-wave 24 theory. This appears justified given the finite, although strongly reduced, staggered moment obtained within the QMC simulations. As translational symmetry is broken in each realization for the disordered system, we adopt a a real-space formulation of the spin-wave approach to finite systems. In the following, we use the method of Ref. 25], which is based upon solving a non-hermitian eigenvalue problem and subsequent orthogonalization to obtain the bosonic eigenmodes of the quadratic part of the spin-wave Hamiltonian. Similar approaches have been used recently to study site-dilution effects in the Heisenberg model on the square 26] and honeycomb 27] lattice. For details on the numerical scheme, we refer to Ref. 25].

We perform the above procedure for typically 1000 disorder realizations for system sizes $L=8,16,24,32$ and 40 for various values of $\delta$. The spin-wave estimate of $\overline{\langle s(\pi, \pi)\rangle}$ for given values of $L$ and $\delta$ is finally obtained by averaging the local staggered moments from the various disorder realizations. We found that for $\delta>1$ a small fraction of sites attain negative values of the local staggered moment. We treat this artifact of the linear spinwave theory, related to the singular disorder distribution 


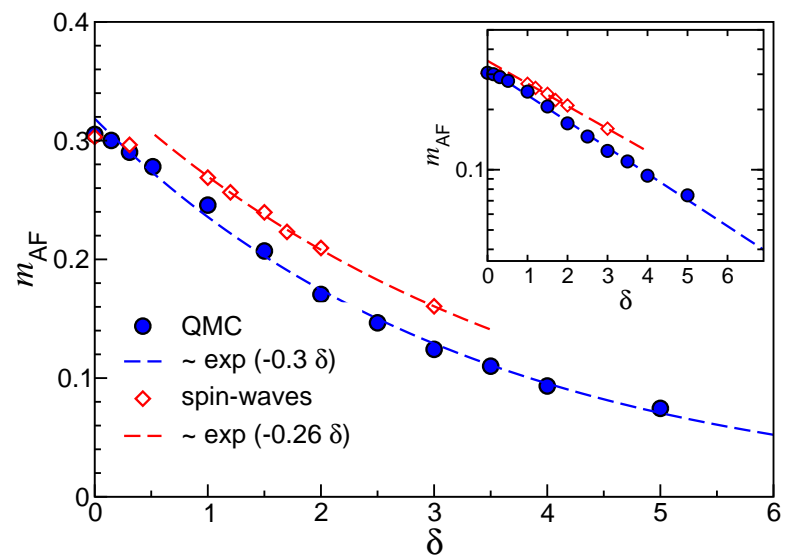

FIG. 3: (Color online) Disorder averaged antiferromagnetic order parameter as a function of the disorder strength $\delta$. Dashed lines denote exponential fits of the data for $\delta>1$, and the inset shows the same data on a semi-logarithmic plot. Note also that error bars are smaller than symbol sizes.

at $J=0$, by explicitly setting the local staggered moments of the corresponding sites to zero before performing the averaging procedure. Results of the SW calculation are shown in Fig. 2 for various disorder strengths as functions of $1 / L$. Extrapolations to the thermodynamic limit have been performed using third order polynomial fits in $1 / L$, where again high-order terms become important as the randomness increases.

Based on our finite size scaling analysis, we now consider the behavior of the staggered magnetization $m_{\mathrm{AF}}$ in the presence of bond-randomness. We plot the values obtained from both the ED and QMC and the SW calculations as functions of $\delta$ in Fig. [3]

We find the Néel order is very robust against bounded bond randomness. While the SW approach slightly overestimates the order parameter (as expected), the form of the $\delta$-dependence of $m_{\mathrm{AF}}$ calculated within SW theory agrees well with the ED and QMC results. Using the distribution of Eq. (3), the order parameter shows an exponential decay (c.f. the inset Fig. B) of the form

$$
m_{\mathrm{AF}} \sim \exp \left(-c_{m} \delta\right),
$$

with $c_{m}=0.301(5)$ obtained from the ED and QMC calculations, and $c_{m}=0.260(5)$ within SW theory. This suggests that the robust LRO vanishes only in the limit of infinite randomness, i.e. for $\delta \rightarrow \infty$.

In addition to the staggered magnetization, the ordered nature of the Heisenberg antiferromagnet is accompanied by a finite value of the spin stiffness, defined as the second derivative of the GS energy with respect to a twist angle $\Phi$ introduced at the boundary along a direction perpendicular to the order parameter:

$$
\rho_{s}=\left.\frac{3}{2} \frac{\partial^{2} E_{0}}{\partial \Phi^{2}}\right|_{\Phi=0} .
$$

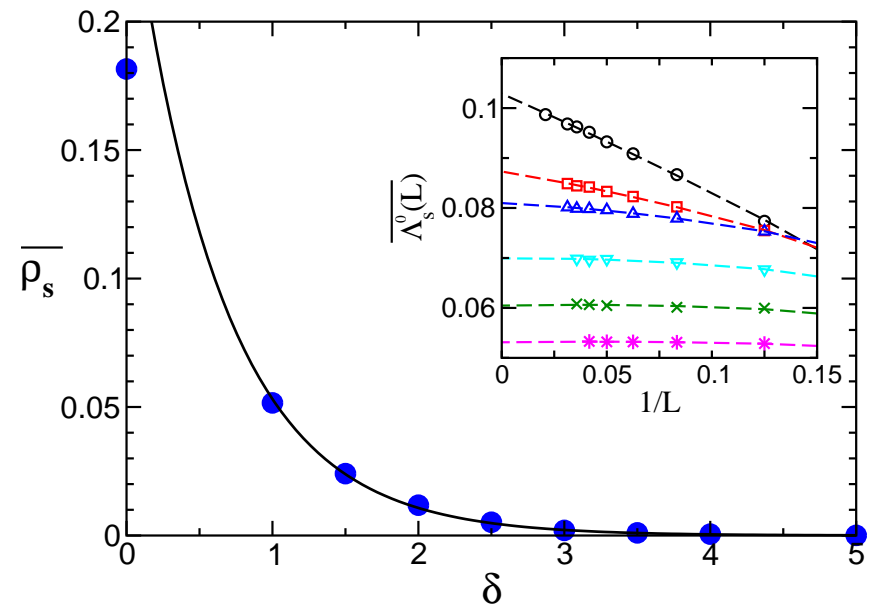

FIG. 4: (Color online) Disorder averaged values of the spin stiffness $\overline{\rho_{s}}$ obtained from QMC simulations on systems with up to $L=32$ and carefull finite size scaling to the thermodynamic limit (see text). The solid line denotes an exponential fit Eq. (7). Inset: Finite size scaling of the disorder average $\omega=0$ current correlator $\overline{\Lambda_{s}^{0}(L)}=-\overline{e_{0}(L)} / 3-2 \overline{\rho_{s}(L)} / 3$ plotted vs $1 / L$ for $\delta=0,1,2,3,4,5$ (different symbols from top to bottom). Dashed lines are quadratic fits. Error bars are smaller than symbol sizes.

In contrast to $m_{\mathrm{AF}}$, the spin stiffness is a dimensionful quantity, and in the clean case, where $\rho_{s}=0.175(2) \mathrm{J}$, scales proportional to the exchange constant $J$. A naive expectation would suggest that in the disordered case, $\rho_{s}$ scales with the averaged value of the exchange, $\bar{J} \sim \frac{1}{\delta+1}$, leading to an algebraic decay for large values of $\delta$. In the following, we show that instead $\rho_{s}$ shows an exponential decay, similar to the staggered magnetization, $m_{\mathrm{AF}}$. From the QMC simulations, $\rho_{s}$ can be evaluated for each disorder realization by measuring the world-line winding number fluctuation [8], and finally performing a disorder averaging. In a clean system, a twist $\Phi$ enforced at the boundaries will be equally distributed among the bonds along the corresponding direction, resulting in a homogeneous twist in the order parameter of $\Phi / L$ per bond. In contrast, for a bond-disordered system, larger twists occur for weaker bonds 28], resulting in an inhomogeneous distribution of the local twist angle. The winding number however, being a global quantity, provides an estimate for the global rigidity of the system under such a twist in the boundary conditions.

The spin stiffness can be expressed as $\rho_{s}=-\frac{e_{0}}{2}-\frac{3 \Lambda_{s}^{0}}{2}$, in terms of GS energy per site without twist, $e_{0}$, and $\Lambda_{s}^{0}$, the $\omega=0$ current-current correlation function $\Lambda_{s}(\omega)=$ $\frac{1}{L^{2}} \int_{0}^{\beta} d \tau \mathrm{e}^{-i \omega \tau}\left\langle j_{s}(\tau) j_{s}(0)\right\rangle$ 8, 30]. According to spinwave calculations [29, 30], in the clean case $\Lambda_{s}^{0}(L)$ scales like $\Lambda_{s}^{0}(L)=\Lambda_{s}^{0}+\frac{a_{1}}{L}+\frac{a_{2}}{L^{2}}+\ldots$. whereas the GS energy scales as $e_{0}(L)-e_{0} \sim \frac{1}{L^{3}}[8,31]$. We find that the $1 / L^{3}$ scaling of $e_{0}(L)$ also holds for the disordered aver- 
aged values. To reliably obtain the disorder average spin stiffness $\overline{\rho_{s}}$ in the thermodynamic limit, we subtracted the energy contribution to the stiffness and performed polynomial fits in $1 / L$ for the disorder averaged values of $\overline{\Lambda_{s}^{0}(L)}$, as shown in the inset of Fig. 4 Interestingly, for increasing disorder, finite size effects get strongly reduced which implies that the thermodynamic limit value for $\overline{\rho_{S}}$ is, within error bars, reached for smaller systems as disorder increases. Since more QMC steps are needed for each random sample to compute the fluctuations in the winding numbers, than for the structure factor, the disorder average was performed over slightly less random samples. Typically 1000 samples were used for the smaller and 200 for the largest lattice with $L=32$. Our results for $\overline{\rho_{s}}$ as a function of $\delta$ are shown in Fig. 4 We find that the spin stiffness remains finite upon increasing the disorder, showing an exponential reduction with $\delta$, similar to the $\mathrm{AF}$ order parameter. In fact, a decay

$$
\rho_{s}(\delta) \sim \exp \left(-c_{\rho} \delta\right)
$$

with $c_{\rho}=1.60(2)$, fits the QMC data for $\delta>1$.

In conclusion we studied the ground state properties of the bond-disordered spin-1/2 Heisenberg model on the square lattice, and found the antiferromagnetic order to be rather robust against bond-randomness: Both the staggered magnetization and the spin stiffness get reduced exponentially with $\delta$, but vanish only in the limit of infinite randomness. These observations suggest a reduced relevance of disorder effects on quantum Heisenberg models in dimensions $D>1$. Indeed, and in contrast to the Ising model in a transverse field, where disorder is relevant also for $D>1$ [32, 33], the infinite randomness fixed point is unstable in XY and Heisenberg AFMs for $D \geq 2[19]$. This result of a renormalization group study is confirmed here for the Heisenberg case on the square lattice. It would be interesting to achieve an analytical understanding of the exponential decay we obtained for both the order parameter and the spin stiffness. In an earlier study, performed for a similar random-exchange AFM, a finite critical disorder strength was found to destroy Néel order and to drive the system into a paramagnetic phase 34]. Based on simulations using significantly larger system sizes at essentially zero temperature, we conclude on the absence of any such finite critical disorder strength. We furthermore expect a similar robustness of Néel-ordered GSs of quantum AFMs on other bipartite lattices, such as the honeycomb lattice. In contrast, for the case of a triangular lattice the interplay between randomness and frustration is expected to destroy the ordered GS of the pure case [35], and drive the system into a spin-glass phase for strong bond-disorder [19]. Finally, the question arises, how the excitation spectrum and finite temperature properties are affected by bond-disorder. Preliminary results 36] indicate that upon increasing the disorder strength, the character of the low-energy excitations changes from extended spin waves to localized spin-cluster flips.

We acknowledge valuable discussion with A. Sandvik, Y.-C. Lin and F. Iglói. The research of N.L. was supported by NSERC of Canada, and the numerical simulations were carried out on the WestGrid network and at NIC Jülich.

[1] D. A. Huse, Phys. Rev. B 37, 2380 (1988).

[2] J. D. Reger and A. P. Young, Phys. Rev. B 37, 5978 (1988).

[3] S. Chakravarty, B. I. Halperin, and D. R. Nelson, Phys. Rev. Lett. 60, 1057 (1989); Phys. Rev. B 39, 2344 (1989).

[4] R. R. P. Singh, Phys. Rev. B 39, 9760 (1989).

[5] E. Manousakis, Rev. Mod. Phys. 63, 1 (1991).

[6] H. J. Schulz, T. A. L. Ziman and D. Poilblanc, J. Phys. I 6, 675 (1996).

[7] B. B. Beard and U.-J. Wiese, Phys. Rev. Lett. 77, 5130 (1996).

[8] A. W. Sandvik, Phys. Rev. B 56, 11678 (1997).

[9] R. J. Birgeneau, M. Greven, M. A. Kastner, Y. S. Lee, B. O. Wells, Y. Endoh, K. Yamada and G. Shirane, Phys. Rev. B 59, 13788 (1999); R. Coldea, S. M. Hayden, G. Aeppli, T. G. Perring, C. D. Frost, T. E. Mason, S.-W. Cheong and Z. Fisk, Phys. Rev. Lett. 86, 5377 (2001).

[10] O. P. Vajk, P. K. Mang, M. Greven, P. M. Gehring and J. W. Lynn, Science 295, 1691 (2002).

[11] K. Kato, S. Todo, K. Harada, N. Kawashima, S. Miyashita and H. Takayama, Phys. Rev. Lett. 84, 4204 (2000). See also A. W. Sandvik, Phys. Rev. Lett. 86, 3209 (2001); S. Todo, H. Takayama and N. Kawashima, Phys. Rev. Lett. 86, 3210 (2001).

[12] A. W. Sandvik, Phys. Rev. B 66, 024418 (2002).

[13] C. Doty and D. S. Fisher, Phys. Rev. B 45, 2167 (1992).

[14] D. S. Fisher, Phys. Rev. B 50, 3799 (1994).

[15] F. Iglói, R. Juhász and H. Rieger, Phys. Rev. B 61, 11552 (2000).

[16] N. Laflorencie and H. Rieger, Phys. Rev. Lett. 91, 229701 (2003).

[17] E. Orignac and T. Giamarchi, Phys. Rev. B 57, 5812 (1998).

[18] R. Melin, Y.-C. Lin, P. Lajk, H. Rieger and F. Igli, Phys. Rev. B 65, 104415 (2002); E. Yusuf and K. Yang, Phys. Rev. B 65, 224428 (2002).

[19] Y.-C. Lin, R. Melin, H. Rieger and F. Igli, Phys. Rev. B 68, 024424 (2003).

[20] Y.-C. Lin, H. Rieger, and F. Iglói, unpublished.).

[21] The disorder strength $\delta$ can be defined as the variance of a random variable $\ln J$ which in case of a flat distribution is related to $W$ according to $\delta^{2}=1-\frac{1-W^{2}}{4 W^{2}}\left[\ln \left(\frac{1+W}{1-W}\right)\right]^{2}$.

[22] O. F. Syljuasen and A. W. Sandvik, Phys. Rev. E 66, 046701 (2002).

[23] Note the value obtained in Ref. [8] using an unconstrained finite size scaling fit is $0.3062(6)$ and $0.3070(3)$ with a constrained fit.

[24] K. Yosida, Theory of Magnetism (Springer, 1996).

[25] S. Wessel and I. Milat, Phys. Rev. B 71, 104427 (2005).

[26] E. R. Mucciolo, A. H. Castro Neto, and C. Chamon, Phys. Rev. B 69, 214424 (2004).

[27] E. V. Castro, et al., cond-mat/0508204

[28] A. Paramekanti, N. Trivedi and M. Randeria, Phys. Rev. B 57, 11639 (1998).

[29] R. R. P. Singh and D. Huse, ibid. 40, 7247 (1989). 
[30] T. Einarsson and H. J. Schulz, Phys. Rev. B 516151 (1995).

[31] H. Neuberger and T. Ziman, Phys. Rev. B 39, 2608 (1989); D. S. Fisher, Phys. Rev. B 39, 11783 (1989).

[32] C. Pich, A. P. Young, H. Rieger and N. Kawashima, Phys. Rev. Lett. 81, 5916 (1998).

[33] O. Motrunich, S.-C. Mau, D. A. Huse and D. S. Fisher,
Phys. Rev. B 61, 1160 (2000).

[34] A. W. Sandvik and M. Vekic, Phys. Rev. Lett. 74, 1226 (1995).

[35] B. Bernu, C. Lhuillier, and L. Pierre, Phys. Rev. Lett. 69, 2590 (1992).

[36] A. Läuchli, S. Wessel, N. Laflorencie, H. Rieger, unpublished. 